\title{
Influenza vaccination: do the aged reap the benefit?
}

\author{
R Morgan, D King, CJ Turnbull
}

\begin{abstract}
Summary
An audit of 100 elderly in-patients with appropriate medical conditions revealed that $63 \%$ had not been vaccinated this winter. $74 \%$ would have accepted vaccination if offered. A general practitioner questionnaire found that $42 \%$ relied on elderly patients coming forward and requesting vaccination. Unfortunately, many elderly patients are still missing out on influenza vaccination.
\end{abstract}

Keywords: elderly, influenza vaccination

\section{Introduction}

Influenza epidemics can be disasterous, especially for the elderly. The 1989-90 epidemic killed an estimated 26000 people over a 12-week period, most of whom were elderly with chronic medical conditions. ${ }^{1,2}$

Admissions to hospital and deaths associated with pneumonia and influenza can be significantly reduced by the influenza vaccination. ${ }^{3}$ Each autumn, general practitioners valiantly mount an influenza campaign aimed at those groups for whom the influenza vaccination is currently recommended (see box). ${ }^{4}$ Unfortunately, there is a need for improved targetting, since less than $50 \%$ of elderly at risk patients receive vaccination. ${ }^{5}$

We report the results of a survey of general practitioners' views on the influenza vaccination, and an audit of 100 elderly in-patients.

\section{Methods}

Approval was obtained from the Arrowe Park Hospital Ethics Committee.

We sent a postal questionnaire (available from the authors) to 173 general practitioners employed by the Family Health Services Authority in the Wirral area (see box on next page). No attempt was made to obtain information from non-responders.

Arrowe Park Hospital Wirral, Merseyside, UK

R Morgan

D King

CJ Turnbull

Correspondence to

R Morgan MRCIP, Arrowe Park Hospital, Arrowe Park Road, Wirral, Merseyside L49 5PE, UK
An audit of 100 elderly in-patients (mean age 82.1 years; 69 females, 31 males) on five acute geriatric wards who had a medical condition (table) for whom the influenza vaccination is currently recommended, was carried out by means of a structured interview by a doctor unknown to the patient (RM) during December 1993-February 1994.

All patients were mentally competent, (having scored $\mathbf{8}$ or more on an abbreviated mental test $\left.{ }^{6}\right)$, lived at home and were convalescent awaiting discharge. All patients were on the lists of the general practitioners to whom the postal questionnaire was sent.

\section{Results}

The response rate to the questionnaire from general practitioners was $58 \%$. A minority of general practitioners $(11 \%)$ reported that they had experienced difficulty in identifying elderly at-risk patients. As to ensuring vaccination of elderly patients at risk, $47 \%$ of the general practitioners sent out letters and asked patients to call in for vaccination. A few $(11 \%)$ asked the practice nurse to call on patients in their own homes whilst $42 \%$ relied on the media and notices in the surgery to encourage patients to come forward.

The majority $(73 \%)$ of general practitioners thought it would be helpful if hospital doctors mentioned in their discharge letters any elderly at-risk patients who should be vaccinated.

Most general practitioners $(85 \%)$ reported that they had no reservations about the influenza vaccine. The majority $(63 \%)$ thought it was feasible to vaccinate all elderly at-risk patients, although a problem with supply of the vaccine this winter $(1993 / 4)$ had been noted by $77 \%$.

Of the 100 elderly in-patients recruited into this study, the majority $(61 \%)$ had not received the influenza vaccination this winter (table). Most $(74 \%)$ said they would accept an influenza vaccination if offered compared to $17 \%$ who would refuse, due to concern about side effects. A minority $(9 \%)$ were undecided.

General practitioners had never advised an influenza vaccination in $56 \%$ of cases. Most patients $(98 \%)$ had not been asked or advised about the influenza vaccine by a hospital doctor during their admission. Of those patients who had received the influenza vaccine this year, $10 \%$ had initiated vaccination themselves by actively requesting it from their general practitioners. 


\section{Questionnaire}

- Have you experienced any difficulty in identifying elderly patients who should be vaccinated against influenza?

- How do you ensure vaccination of elderly patients at risk?

- Do you have any reservations about the influenza vaccination?

- Do you think it is feasible to vaccinate all of the elderly at risk patients?

- Have you experienced any difficulty in supplying the influenza vaccine due to lack of supplies?

- Do you think it would be helpful if hospital doctors, having identified at-risk elderly patients who have not been vaccinated, mentioned it on the discharge letter?

Table 1 Influenza vaccination: in-patient data

\begin{tabular}{|c|c|c|c|c|c|}
\hline \multirow[b]{2}{*}{ Diagnosis } & \multicolumn{2}{|c|}{$\begin{array}{l}\text { Influenza vaccination } \\
\text { this winter } \\
\end{array}$} & \multicolumn{3}{|c|}{$\begin{array}{l}\text { If offered vaccination, } \\
\text { would you accept it? }\end{array}$} \\
\hline & yes & no & yes & no & don't know \\
\hline $\begin{array}{l}\text { Chronic obstructive } \\
\text { airway disease }\end{array}$ & 23 & 30 & 45 & 4 & 4 \\
\hline $\begin{array}{l}\text { Congestive cardiac } \\
\text { failure }\end{array}$ & 6 & 22 & 14 & 10 & 4 \\
\hline Diabetes mellitus & 10 & 9 & 15 & 3 & 1 \\
\hline$n$ & 39 & 61 & 74 & 17 & 9 \\
\hline
\end{tabular}

\section{Discussion}

Elderly patients with chronic medical conditions are a vulnerable group during influenza epidemics. ${ }^{7}$ Since vaccination against influenza can reduce deaths, ${ }^{3}$ it is logical that this group should be targetted for vaccination. The fact that many elderly at-risk patients are not being vaccinated (61\% in this study), would suggest that ways of improving targetting need to be addressed.

Although most general practitioners had no difficulty in identifying elderly at-risk patients they believed that targetting could be accentuated by hospital doctors mentioning in discharge letters those patients who should receive vaccination. Since influenza immunisation is only offered for two to three months of the year, recommendation in discharge letters at other times should be added to an influenza vaccination data base.

Nguyen-Van-Tam et al reported that whilst $92.5 \%$ of practices actively promoted influenza vaccination, the most frequently used promotional method was a poster displayed in the surgery. ${ }^{8}$ The fact that $42 \%$ of general practitioners in this study do not contact patients individually for vaccination, means effectively that many elderly patients with an appropriate medical condition are not being reached, since many in this group are housebound and would not be able to attend the surgery.

1 Ashley J, Smith T, Dunnell K. Deaths in Great Britain associated with the influenza epidemic of 1989-1990. Popu Trends 1991; 65: 16-20.

2 Nguyen-van-Tam JS, Nicholson KG. Influenza deaths in Leicestershire during the 1989-1990 epidemic: implication for prevention. Epidemiol Infect 1992; 108: 537-45.

3 Barker WH, Mulloolly JP. Influenza vaccination of elderly persons: reduction in pneumonia and influenza of elderly tions and deaths. $\mathscr{F} A M A$ 1980; 244: 2547-9.

4 Joint Joint Committee on Vaccination and Immunisation. disease. London: HMSO, 1990.
In this study the $58 \%$ response rate to the general practitioner questionnaire may have biased the results, although it is unlikely that non-responders would be more active campaigners for the influenza vaccination than responders.

The small in-patient sample (100), does not tell us how big the problem of non-vaccination is in the community, nor how effective the general practitioners were being, only a community study could do that.

When a child is admitted to hospital it is a routine part of taking a history to include details of vaccination status. It is appropriate to apply this to elderly at-risk patients and advise them and their general practitioners that they would benefit from receiving the influenza vaccination each winter. Hospital doctors should not miss the opportunity for health education for in-patients and patients seen in geriatric out-patient clinics, and should actively promote the influenza vaccination.

Elderly patients with a chronic medical condition for whom vaccination is recommended but who are unable to attend the surgery for vaccination should be vaccinated at home by the practice nurse.

These measures would increase the number of older patients receiving the vaccine.

The ultimate benefit to the elderly in terms of reduced hospital admission rates and mortality offsets the initial cost of vaccine supply and practice nurse time. The nature of the influenza virus is such that further epidemics can be anticipated. The number of fatalities ensuing from an influenza epidemic could be reduced by the simple expedience of ensuring increased uptake of vaccination.

The elderly at risk should not be deprived of the benefit of the influenza vaccination (see box).

\begin{tabular}{l} 
Recommendations for \\
improving uptake of influenza \\
vaccination in the elderly \\
\hline include inquiry about previous vaccination in \\
the routine history taking \\
- mention the need for vaccination in hospital \\
discharge summaries or clinic letters \\
vaccination at home for those who are \\
immobile or housebound
\end{tabular}

The authors would like to thank all of the general practitioners in the Wirral area who participated in the postal questionnaire.

5 Nicholson KG. Immunisation against influenza among people aged over 65 living at home in Leicestershire during winter 1991-2. BMF 1993; 306: 974-6.

6 Qureshi KN, Hodkinson HM. Evaluation of a ten question mental test in the institutionalised elderly. Age Ageing 1974; 3: $152-7$.

7 Barker WH, Mulloolly JP. Pneumonia and influenza death during epidemics: implications for prevention. Arch Intern Med 1982; 142: 85-9.

8 Nguyen-Van-Tam J, Nicholson K. Influenza immunisation: policies and practices of general practitioners in England, policies and practices of general practitioners
$1991-1992$. Health Trends 1993; 25: 101-5. 